\title{
La Seguridad Alimentaria como factor sostenible de Paz y Seguridad Internacionales ${ }^{1}$
}

\section{Food Security as a Sustainable Factor of International Peace and Security}

\author{
Adriana Fillol ${ }^{2}$ \\ Universidad de Sevilla (España)
}

Recibido: 30-01-19

Aprobado: 25-03-19

\section{Resumen}

La paz y la seguridad internacionales no sólo son el resultado de la ausencia de guerras, es decir, no son únicamente el fundamento de la preservación del orden o ausencia de violencia, sino que incluyen también, para ser alcanzadas, aspectos diversos, tales como la seguridad alimentaria.

Dentro del abanico de los nuevos riesgos y amenazas a la paz y seguridad internacionales, cada vez más se está teniendo en consideración las repercusiones negativas que tienen las crisis alimentarias y el hambre que sufren en torno a 800 millones de personas en todo el mundo.

La preocupación y abordaje de estos temas hizo que por primera vez, el 30 de marzo de 2016, la FAO se dirigiese al Consejo de Seguridad con el objetivo de reafirmar la necesidad de mejorar la seguridad alimentaria como aspecto fundamental para la consolidación de una paz y seguridad internacional sostenibles.

Palabras-clave: Seguridad Alimentaria, Paz y Seguridad Internacionales, Conflictos, Naciones Unidas.

\footnotetext{
${ }^{1}$ Trabajo realizado en el marco del proyecto de investigación "Los desafíos de los derechos humanos ante los nuevos riesgos y amenazas" (EUIN2017-85437), Programa Estatal de 1+D+I Orientada a los Retos de la Sociedad, Acciones de Dinamización: Europa Investigación 2017.

2 (afillol@us.es) Doctora en Derecho por la Universidad de Sevilla. Centra sus investigaciones en la relación que existe entre la seguridad alimentaria y la paz. 


\begin{abstract}
International peace and security are not only the result of the absence of wars, in other words, they are not only the foundation of the preservation of order or absence of violence, but also include, to be achieved, diverse aspects, such as the food security.

Inside a wide range of new risks and threats to international peace and security, the negative repercussions of the food crises and hunger suffered by 800 million people around the world are increasingly being taken into consideration.

The concerns about these issues meant that for the first time, on March 30, 2016, FAO addressed the Security Council with the objective of reaffirming the need to improve food security as a fundamental aspect for the consolidation of sustainable international peace and security.
\end{abstract}

Key-words: Food Security, International Peace and Security, Conflicts, United Nations.

\title{
1. Introducción: Concepto consolidado de seguridad alimentaria en el plano internacional
}

La interrelación entre la seguridad alimentaria y la paz y seguridad internacionales sirvió de base para la concesión del Premio Nobel de la Paz, en 1949, a Boyd Orr, primer Director General de la FAO. Según Boyd Orr, "el hambre se encuentra en el corazón de los problemas del mundo y a menos que la gente se alimente, los mejores tratados pueden quedar en nada. Las personas hambrientas no pueden ser satisfechas con nada más que con comida"3.

Aunque la definición de seguridad alimentaria ya es de por sí compleja, es necesario señalar que el concepto traducido al español como seguridad alimentaria engloba en realidad a dos dimensiones que esta presenta y que acogen en inglés dos definiciones separadas: "food security", referida fundamentalmente a la cantidad de alimentos disponibles, así como al acceso a los mismos, y "food safety", referida a la calidad o inocuidad de los alimentos.

El concepto de seguridad alimentaria, objeto de un creciente interés académico, jurídico y político, ha experimentado una evolución en su contenido. En efecto, el concepto de seguridad alimentaria no ha tenido un significado unívoco ni estático desde que empezó a emplearse allá por el año 1974 (fecha

\footnotetext{
${ }^{3}$ Véase Boyd Orr, J., "Science, Politics and Peace", Lecture delivered on the occasion of the award of the Nobel Peace Prize of 1949. Cita encontrada en el discurso que el actual Director de la FAO, José Graziano da Silva, ofreció en 2016 ante el Consejo de Seguridad durante la Reunión de fórmula Arria sobre Seguridad Alimentaria, Nutrición y Paz, disponible en: http://www.fao.org/about/who-we-are/ director-gen/faodg-statements/detail/en/c/404723/, consultado el 15 de enero de 2019.
} 
de la primera Conferencia Mundial de la Alimentación), sino que más bien ha presentado una ampliación de su contenido (extendiendo su alcance, sus dimensiones e incluyendo elementos tanto cuantitativos - de cantidad-, como cualitativos -de calidad-), hasta llegar al actual concepto que propuso la Organización de las Naciones Unidas para la Alimentación y la Agricultura (FAO) en 1996, que es el que cuenta con una mayor aceptación en el marco internacional.

Si bien el actual concepto de seguridad alimentaria ha sido auspiciado y promovido por la FAO, este concepto se ha venido reiterando (sobre todo a partir de 1996) en los diferentes planes y declaraciones que se adoptan tras la celebración de las Cumbres Mundiales de la Alimentación. En estas Cumbres los Estados participan y se manifiestan a través de su representantes (Jefes de Estado y de Gobierno), por tanto, podemos considerar que el actual concepto de seguridad alimentaria, aunque fue elaborado por la FAO, también ha sido fruto del consenso de los Estados; consenso que se manifiesta en esos planes y declaraciones que se adoptan tras las Cumbres y donde se reitera el concepto de seguridad alimentaria que promovió la FAO.

En este sentido, el actual concepto de seguridad alimentaria, fruto de la Cumbre Mundial sobre la Alimentación, que se celebró en Roma del 13 al 17 de noviembre de 1996, queda formulado de la siguiente manera:

\footnotetext{
"Existe seguridad alimentaria cuando todas las personas tienen en todo momento acceso físico y económico a suficientes alimentos inocuos y nutritivos para satisfacer sus necesidades alimenticias y sus preferencias en cuanto a los alimentos a fin de llevar una vida activa y sana"4.
}

Esta definición incluye en su formulación elementos cualitativos (nutritivos, de calidad o inocuidad de los alimentos) y no sólo elementos cuantitativos como hasta entonces se había hecho ${ }^{5}$. Sin embargo, en relación a este trabajo nosotros nos vamos a centrar únicamente en la dimensión cuantitativa (disponibilidad y acceso a los alimentos) de la seguridad alimentaria y su interrelación con la paz y seguridad internacionales.

\footnotetext{
${ }^{4}$ Plan de Acción de la Cumbre Mundial sobre la Alimentación, Roma, 1996, apartado primero.

${ }^{5}$ Afifi, A., "Sources et norms de sécurité alimentaire en Droit International", en Mahiou A., and Snyder. F., (Ed.), Food Security and Food Safety/ La Sécurité Alimentaire, Martinus Nijhoff Publishers, Dordrecht, Netherlands, 2006, p.93.
} 


\section{Hacia un marco amplio y multidimensional del mantenimiento de la paz y seguridad internacionales}

Los cambios estructurales que se están produciendo en la sociedad internacional globalizada han dado como consecuencia la emergencia de nuevos riesgos y amenazas que afectan a la seguridad humana y a la paz y seguridad internacionales ${ }^{6}$. Hoy en día el marco jurídico que regula el mantenimiento de la paz y seguridad internacionales se encuentra ante nuevos riesgos y amenazas, diferentes de aquellos para los que se creó ${ }^{7}$.

El surgimiento y/o profundización de nuevos fenómenos, actores y fuerzas transnacionales como la pobreza, el cambio climático, el hambre, las enfermedades infecciosas, el flujo masivo de personas o la escasez de recursos naturales respecto a la explosión demográfica mundial, han alterado significativamente las perspectivas sobre el mantenimiento de la paz y seguridad internacionales, así como las causas, dinámicas y consecuencias de los conflictos y las crisis humanitarias asociadas. Los conflictos se han vuelto más complejos y los elementos que los definen están más fragmentados.

A su vez, las nuevas expresiones de amenazas están relacionadas entre sí, y la forma idónea y acertada de afrontarlas debe darse simultáneamente a nivel global, regional y nacional ${ }^{8}$. En palabras de Del Arenal, "la seguridad nacional y la seguridad internacional están totalmente interrelacionadas". Según Toucoz, "la seguridad nacional y la seguridad internacional se han ido interrelacionando crecientemente; la seguridad no es divisible ya en distintos aspectos, ni por segmentos de actividad, como el militar, el económico, el social, el político, ni por ámbitos territoriales, como el nacional y el internacional" ${ }^{10}$.

Por ejemplo, especialmente el cambio climático ha sido identificado durante la última década como un factor emergente multiplicador de las amenazas a la seguridad internacional ${ }^{11}$ y la estabilidad social, económica y política de los Estados. El cambio climático incide negativamente en la producción agrícolaganadera, en la frecuencia e intensidad de las precipitaciones, en la acidificación de las aguas de los océanos, en la desaparición de la cobertura vegetal y, en definitiva,

\footnotetext{
${ }^{6}$ Madrueño-Aguilar, R., "Human Security and the New Global Threats: Discourse, Taxonomy and Implications", Global Policy, vol. 7, no 2, 2016, pp. 156-173.

7 UN.Doc. S/PV.7389, 23 February 2015, "Maintenance of international peace and security", p.54.

${ }^{8}$ Laborie Iglesias M, "La Evolución del Concepto de Seguridad", Documento Marco 05/2011, Instituto Español de Estudios Estratégicos, Ministerio de Defensa, 2011, p.2.

9 Del Arenal, C., "El nuevo escenario mundial y la teoría de las relaciones internacionales", en Pérez González, M. (coord.), Hacia un nuevo orden internacional y europeo: estudios en homenaje al profesor don Manuel Diez de Velasco, Tecnos, Madrid, 1993, p. 80.

10 Toucoz, J., "Mondialisation et sécurité économique internationale. Quelques remarques juridiques et institutionnelles", RGDIP, n 3, pp. 623-624.

${ }^{11}$ Giles Carnero, R., "El cambio climático como riesgo y amenaza para la seguridad: derivaciones en el desarrollo del régimen jurídico internacional en materia de clima", Araucaria. Revista Iberoamericana de Filosofia, Política y Humanidades, vol. 18, núm. 36, 2016, pp. 315-338.
} 
en la vida del ser humano. Ello conlleva riesgos y amenazas bien visibles ya en la actualidad, poniendo en tela de juicio, incluso, la propia alimentación del ser humano. Pensemos también en los actuales flujos masivos involuntarios de personas, es decir, los provocados por las guerras, los conflictos étnicos, el hambre, las adversidades climáticas o la falta de agua, que pueden generar tensiones, inestabilidad o conflictos tanto en las zonas de tránsito como en las destino.

En este sentido, van a entrar en acción en el plano internacional problemas o desafíos nuevos que pueden afectar potencialmente (riesgo) o de hecho (amenaza) a la paz y seguridad internacionales -a pesar de que se produzcan dentro de las fronteras de un Estado-, y que pueden dar lugar a su calificación, por parte del Consejo de Seguridad, como una amenaza a la paz y seguridad internacionales. De hecho, la práctica del Consejo ha ido ampliando el concepto de amenaza a la paz y seguridad internacionales y lo ha extendido más allá de los tradicionales conflictos armados inter-estatales. En palabras de Abellán Honrubia, "hemos asistido a una ampliación progresiva del concepto de mantenimiento de la paz y seguridad internacionales por parte del Consejo de Seguridad de las Naciones Unidas, mediante una interpretación extensiva de los supuestos de amenazas a la paz y seguridad internacionales" 12 . Si esto es así, es obvio decir que está abierta la posibilidad de que se pueda calificar como una amenaza a la paz y seguridad internacionales a una hambruna generalizada o grave aunque se produzca en el interior de un Estado.

\subsection{La multidimensionalidad de la seguridad}

Desde finales de la guerra fría, la seguridad ha sido objeto de investigación por diferentes autores, entre los que destaca el Profesor Buzan, que hace referencia expresa a la multidimensionalidad de la seguridad en el entendido de que esta se compone de cinco dimensiones que están interrelacionadas: la militar, la política, la económica, la social y la medioambiental ${ }^{13}$. Siguiendo esta misma línea, Barbé considera que "en la búsqueda de soluciones al problema de la inseguridad, los Estados deben afrontar cada vez con mayor frecuencia circunstancias fuera de su control, como crisis económicas estructurales y tendencias económicas, demográficas, ambientales y financieras a las que sólo se puede dar solución mediante acciones comunes y solidarias"14. Podemos afirmar que actualmente se descarta la idea de que la seguridad es exclusivamente una función del poder nacional o de la fuerza militar.

\footnotetext{
${ }^{12}$ Abellán Honrubia, V., "Sobre el método y los conceptos del Derecho Internacional Público", en Soberanía del Estado y Derecho Internacional: Homenaje al Profesor Juan Antonio Carrillo Salcedo (O.C.), primer volumen, Universidad de Sevilla, Secretariado de Publicaciones, 2005, p.67.

${ }^{13}$ Buzan, B., "New Patterns of Global Security in the Twenty-First Century", International Affairs, Vol. 67, No3, 1991, p. 433

${ }^{14}$ Barbé, E., Relaciones Internacionales, Tecnos, Madrid, 1995, p. 279.
} 
Esta revalorización de la multidimensionalidad de la seguridad ya fue introducida también en el texto aprobado en 1987 en la Conferencia de Naciones Unidas en relación al Desarme y el Desarrollo, según el cual: "la seguridad tiene no sólo aspecto militares, sino también aspectos económicos, sociales, humanitarios y de derechos humanos, y ecológicos" ${ }^{15}$. Paralelamente a la revalorización de la multidimensionalidad de la seguridad, en la década de los setenta, con la aparición de la investigación para la paz como disciplina, la doctrina avanzó hacia un concepto más amplio de paz. Frente a la paz negativa, entendida como la ausencia de violencia, se propuso la paz positiva como un proceso complejo, a medio y largo plazo, que se relaciona con el fortalecimiento de los derechos humanos, el desarrollo y el respeto al medio ambiente.

En efecto, Naciones Unidas viene entendiendo que la paz, la seguridad, los derechos humanos y el desarrollo sostenible están íntimamente relacionados. Prueba de ellos son los Objetivos de Desarrollo Sostenible, en concreto el objetivo $16^{16}$. Asimismo, si nos retrotraemos en el tiempo, la Resolución 377 (V), de 3 de noviembre de 1950, (titulada Unión pro paz) de la Asamblea General mencionaba a todos los propósitos de la Organización cuando afirmaba que: "el mantenimiento de una paz real y duradera depende también del cumplimiento de todos los propósitos y principios enunciados en la Carta de las Naciones Unidas"17.

En este sentido, basta recordar que la promoción y protección de la seguridad alimentaria encaja con los propósitos de Naciones Unidas, de acuerdo con el artículo 55 de la Carta, y, por tanto, habría que tomar en consideración a la seguridad alimentaria como un factor sostenible de paz "real y duradera". El artículo 55 de la Carta de Naciones Unidas dispone que:

\begin{abstract}
“con el propósito de crear las condiciones de estabilidad y bienestar necesarias para las relaciones pacíficas y amistosas entre las naciones [...], la Organización promoverá: a) niveles de vida más elevados, [...] condiciones de progreso y desarrollo económico y social; b) la solución de problemas internacionales de carácter económico, social y sanitario, y de otros problemas conexos [...]; c) el respeto universal a los derechos humanos de todos [...]".
\end{abstract}

De lo dispuesto en este artículo se puede deducir lo siguiente: con el objetivo de crear sociedades pacíficas, la Organización de Naciones Unidas promoverá y protegerá la seguridad alimentaria en la medida en que ello favorece el nivel

${ }_{15}$ Referencia encontrada en Morán Blanco, S. "La seguridad medioambiental y el cambio climático: consecuencias a nivel internacional”, en Azeredo Lopes, J.A., Fernández Sánchez, P.A., Seguridad Medioambiental y Orden Internacional, Atelier, Barcelona, 2015, p.77.

${ }_{16}$ Este Objetivo se centra en promover sociedades pacíficas e inclusivas para el desarrollo sostenible. Véase: UN Doc. A/RES/70/1, 21 de octubre de 2015, "Transformar nuestro mundo: la Agenda 2030 para el Desarrollo Sostenible", p.29.

${ }_{17}^{17}$ UN.Doc, A/RES/377 (V), Unión pro paz, de 3 de noviembre de 1950, para. 14. 
de vida de las personas, las condiciones de progreso y desarrollo, la respuesta a problemas internacionales de carácter económicos, social y sanitario (las crisis alimentarias afectan a la salud de las personas, ya que las sitúa sobre una mayor vulnerabilidad a contraer enfermedades, y además pueden ser consideradas como problemas internacionales de carácter económico y social) y el respeto universal del derecho fundamental a estar protegidos contra el hambre ${ }^{18}$.

Si entendemos que la seguridad ha tomadoun carácter multidimensional (que incluye aspectos militares, políticos, económicos, sociales, medioambientales y humanitarios), resulta adecuado decir que la seguridad alimentaria tiene que tomarse en consideración a la hora de abordar las llamadas Estrategias de Seguridad.

El hecho de que el concepto de seguridad en la actualidad se conciba como un concepto holístico que está conformado por diferentes dimensiones interrelacionadas hace posible que se amplíen las amenazas y los riesgos a los que hay que hacer frente en el plano internacional y que pueden dar lugar a su calificación por el Consejo de Seguridad como amenazas a la paz y seguridad internacionales, como sucedió con el ébola ${ }^{19}$. ¿Por qué no pensar que el hambre o la grave falta de alimentos podrían ser calificadas como una nueva amenaza por parte del Consejo de Seguridad?

\subsection{La consolidación de la paz para alcanzar una paz sostenible}

Es bien conocido que la paz no sólo es el resultado de la ausencia de guerras y de conflictos entre Estados. El Presidente del Consejo de Seguridad de las Naciones Unidas ya lo dijo en su Declaración de 1992:

"La ausencia de guerra y de conflictos militares entre Estados no asegura por sí misma la paz y la seguridad internacionales. Las causas no militares de inestabilidad en las esferas económica, social, humanitaria y ecológica se han convertido en amenazas a la paz y la seguridad" ${ }^{20}$.

Fernández Sánchez, por ejemplo, entre otros, considera que "la paz formal no basta y que ésta debe venir acompañada de una actitud que genere paz estable y duradera"21. Estas cuestiones, desde la Guerra Fría, han hecho que Naciones Unidas haya incluido progresivamente el objetivo de "consolidación

${ }^{18}$ Artículo 11.2 del PIDESC.

${ }^{19}$ UN.Doc., S/RES/2177 (2014), de 18 de septiembre de 2014, p.1, en la que afirma que: [...] "Habiendo determinado que el alcance sin precedentes del brote del ébola en África constituye una amenaza para la paz y la seguridad internacionales [...]".

${ }^{20}$ UN.Doc., S/23500, 31 enero 1992, Nota del Presidente del Consejo de Seguridad, p.3.

${ }^{21}$ Fernández Sánchez, P.A., Operaciones de Mantenimiento de la Paz (Vol.I), Análisis Jurídico de las Operaciones de Mantenimiento de la Paz, Ministerio de Educación y Cultura, Universidad de Huelva, Madrid, 1998, p. 19.

Araucaria. Revista Iberoamericana de Filosofia, Politica, Humanidades y Relaciones Internacionales, año $21, \mathrm{n}^{\circ} 42$. Segundo semestre de 2019. Pp. 157-182. ISSN 1575-6823 e-ISSN 2340-2199 doi: 10.12795/araucaria.2019.i42.08 
de la paz" ${ }^{22}$ en sus estrategias para el mantenimiento de la paz y seguridad internacionales.

La creación de una nueva arquitectura de consolidación de la paz en Naciones Unidas refleja un creciente reconocimiento dentro de la comunidad internacional de los vínculos entre el establecimiento de la paz, las operaciones de mantenimiento de la paz y la consolidación de la paz para alcanzar una paz sostenible. Esto es, de los vínculos entre los derechos humanos ${ }^{23}$, las cuestiones ambientales, el desarrollo o la estabilidad política como aspectos clave para la consecución de una paz duradera. Por ejemplo, en el Informe del entonces Secretario General, Boutros Boutros-Ghali, "Un programa de Paz", de 1992, la consolidación de la paz se presentó como el seguimiento lógico del establecimiento y mantenimiento de la paz con el objetivo de evitar la recaída en el conflicto una vez que se hubiera logrado un acuerdo de paz ${ }^{24}$.

Esta idea de consolidación de la paz ha sido asumida progresivamente tanto por el Consejo de Seguridad como por el Comité Especial de Operaciones de Mantenimiento de la Paz, que han reconocido la importancia de la consolidación de la paz como parte integrante del éxito de las operaciones de mantenimiento de la paz.

El hecho de que la consolidación de la paz requiera de medidas relacionadas con la protección los derechos humanos es una cuestión que el propio Consejo de Seguridad asume. Por ejemplo, en la Resolución de 27 de abril de 2016, el Consejo reconoce expresamente que "el desarrollo, la paz y la seguridad, y los derechos humanos están interrelacionados y se refuerzan mutuamente" 25 , y recuerda también que "la determinación de establecer una paz justa y duradera en todo el mundo de conformidad con los propósitos y principios de la Carta de las Naciones Unidas"26. Hacemos hincapié en esta interrelación entre la consolidación de paz y el respeto de los derechos humanos porque el derecho a la alimentación es un derecho humano reconocido y la seguridad alimentaria favorece y facilita su pleno ejercicio. Por tanto, podemos deducir que si queremos consolidar la paz, a través de la promoción y protección de los derechos humanos (entre otras), es lógico que la seguridad alimentaria deba

${ }^{22}$ La noción de "consolidación de la paz" fue asumida en 2005 en las Resoluciones que adoptaron conjuntamente la Asamblea General y el Consejo de Seguridad en diciembre, Resoluciones A/RES/60/180 y S/RES/1645 (2005), por las que se creó tres entidades con sede en Nueva York etiquetadas como la "Arquitectura de Consolidación de la Paz": la Comisión de Consolidación de la Paz, el Fondo para la Consolidación de la Paz y la Oficina de Apoyo a la Consolidación de la Paz.

${ }^{23}$ Uribe Vargas nos recuerda en su libro esta relación inseparable entre la paz positiva y la protección de los derechos, en el entendido de que "derechos humanos y paz, como se consagró en el manifiesto de Varsovia, son términos equivalentes y complementarios que al no reconocerse, terminarían precipitándonos a la barbarie". Véase: Uribe Vargas, D., El Derecho a la Paz, Facultad de Derecho de la Universidad Nacional de Colombia, Instituto para el desarrollo de la Democracia, Bogotá, 1996, p.248.

${ }^{24}$ Informe contenido en las Resoluciones A/47/277 y S/24111 de 17 de junio de 1992, pp.6-7.

${ }^{25}$ UN Doc., S/RES/2282 (2016)*, de 27 de abril de 2016, p.1.

${ }^{26}$ Ibidem. 
considerarse como una medida de consolidación mediante la que se protege el derecho humano a la alimentación.

En definitiva, en el actual orden internacional, globalizado y con nuevas amenazas emergentes, el propósito de mantener la paz y la seguridad internacionales implica no sólo garantizar una paz formal, entendida como la ausencia de conflictos, sino también llevar a cabo medidas y acciones positivas en aras de alcanzar una paz duradera y sostenible (con un claro enfoque multidimensional), en las que se tengan en cuenta los aspectos económicos, sociales, políticos, medioambientales y la protección de los derechos humanos ${ }^{27}$. En este sentido, las acciones promotoras y protectoras de la seguridad alimentaria resultan ser medidas importantes para el propósito de consolidación de la paz. El acceso y disponibilidad de alimentos es clave para el desarrollo sostenible, la eliminación de la pobreza, el desarrollo económico en los Estados afectados por los conflictos y el respeto de los demás derechos humanos, incluyendo obviamente el respeto y la protección del derecho humano a una alimentación adecuada ${ }^{28}$. Entendemos, pues, que si la población de un determinado lugar no come o no accede a los alimentos, en cantidad y calidad suficiente, de forma estable, difícilmente podrá afirmarse que las medidas de consolidación de paz tengan éxito. En este sentido, el pilar de la paz debe armonizarse con los pilares de la seguridad alimentaria.

\section{La falta de alimentos como causa y efecto de los conflictos}

Para esclarecer el análisis sobre la interrelación entre la seguridad alimentaria con la paz y la seguridad internacionales es necesario, en primer lugar hacer, mostrar cómo la falta de alimentos contribuye a aumentar el riesgo de inestabilidad social y aparición y/o mantenimiento de los conflictos para, posteriormente, hacer referencia a cuáles son las consecuencias a corto, medio y largo plazo de los conflictos sobre la seguridad alimentaria. Si esto es así, es coherente pensar que la protección de la seguridad alimentaria puede contribuir a la prevención de los conflictos a la vez que es un factor clave, que no único, a tener en cuenta en las estrategias de consolidación de la paz que lleve a cabo Naciones Unidas.

Comienzan a realizarse estudios y análisis por parte de la doctrina, y de organismos especializados y programas de Naciones Unidas, sobre cómo la falta de alimentos o la inseguridad alimentaria pueden aumentar el riesgo de conflicto o contribuir a que este se intensifique o prolongue ${ }^{29}$.

27 "Security Council endorses importance of 'Multidimensional' Approach to Peacekeeping aimed at facilitating Peacebuilding, preventing relapse into Conflict”. SC, SC/10888, 21 january 2013.

28 Véase: Ayala, A., "A human rights approach to the health implications of food and nutrition insecurity", Public Health Reviews, 38:10, 2017.

29 Simmons, E., "Harvesting Peace: Food security, conflict, and cooperation", USAID, vol. 14, no 
Hay que entender que los conflictos han evolucionado, son cada vez más diversos y obedecen a diferentes causas. Por ello, no sólo hacemos referencia, en este apartado, a los conflictos armados entre Estados sino también a conflictos civiles internos, con actores no estatales (como rebeldes, grupos organizados, terroristas) que luchan por la independencia, separación o el control político, o a los conflictos comunales en los que no tiene por qué haber un claro objetivo militar, político o ideológico. Como destaca Reno, hay innumerables pequeñas guerras sin líneas de frente o campos de batalla o zonas de conflicto claras, sin distinciones entre combatientes y civiles, y sin ideología ${ }^{30}$. Los nuevos tipos de conflicto no se abordan fácilmente con los instrumentos tradicionales, como la diplomacia o los medios militares, ya que los nuevos actores que intervienen en ellos a menudo se apoyan en una amplia gama de factores, como problemas políticos, económicos, sociales y ambientales de fondo, para aprovecharse de una situación de inestabilidad o descontento social y avanzar en sus propósitos.

En este sentido, Hendrix y Brinkman, consideran en su estudio que la inseguridad alimentaria, promovida por el incremento del precio de los alimentos $^{31}$, los desastres climáticos o la escasez de recursos naturales, puede impulsar, junto a otros factores (como la débil respuesta del Estado en períodos de escasez), los conflictos civiles, los conflictos comunales o incluso el malestar social y el comportamiento violento ${ }^{32}$. Por tanto, estos autores entienden que la falta de alimentos es un potenciador del inicio de conflictos.

\footnotetext{
3, 2013, pp. 18-23.

${ }^{30}$ Reno, W., Warfare in independent Africa, Cambridge University Press, New York, pp.2-30, 2011.

${ }^{31} \mathrm{La}$ inseguridad alimentaria causada por el aumento del precio de los alimentos es una fuente visible de incertidumbre popular y malestar social. Los aumentos repentinos en los precios de los alimentos han exacerbado el riesgo de agitación socio-política y consecuentes conflictos, como se observó, por ejemplo, en Egipto (1977), Marruecos (1981), Túnez (1984) y Jordania (1996). Véase: Breisinger, C. et al., "How to build resilience to conflict: The role of food security", IFPRI Food Policy Report, Washington, 2014.

32 Véase: Hendrix, C., Brinkman, H.J., "Food Insecurity and Conflict Dynamics: Causal Linkages and Complex Feedbacks", Stability: International Journal of Security and Development, 2(2), 2013, p.26.

Por ejemplo, la pobreza, el hambre y la inseguridad alimentaria, junto con una distribución desigual de los ingresos, de las tierras y de otros bienes materiales, pueden crear sentimientos de ira, desesperanza e injusticia entre los diversos grupos de población. Tales agravios pueden ser utilizados por individuos y grupos con el deseo de fomentar la violencia. Véase: Pinstrup-Andersen, P., Shimokawa, S., "Do poverty and poor health and nutrition increase the risk of armed conflict onset?", Food Policy, 33(6), 2008, pp.513-520.Las personas pueden recurrir a la violencia, que en este contexto la literatura considera como un comportamiento antisocial, cuando su seguridad humana (incluida la seguridad alimentaria) se ve amenazada, especialmente cuando no hay instituciones estatales que sean capaces y estén dispuestas a prevenir o hacer frente a tales riesgos. Véase: Blattman, C., Miguel, E., "Civil war", Journal of Economic Literature, 48(1), 2010, pp. 3-45. En este contexto, los estudios también investigan las razones que tienen los individuos para unirse a grupos armados. Entre algunas razones, Hirshleifer, considera que ciertas personas eligen participar y apoyar a los grupos armados porque pueden beneficiarse de su protección en términos de mejores oportunidades económicas, así como de saqueo y apropiación. Véase: Hirshleifer J., The Dark Side of the Force: Economic Foundations of Conflict Theory, Cambridge, Cambridge University Press, 2001.
} 
Cliffe apunta a que en períodos de escasez de alimentos, motivados por diferentes motivos, la desestructuración o fragilidad de las instituciones estatales en la respuesta que ofrezca a la población es lo que diferencia el hecho de que ante mismas situaciones (de escasez de alimentos) algunas den lugar al conflicto y otras no ${ }^{33}$. En concreto, Cliffe entiende que muchos Estados se pueden enfrentar puntualmente al incremento de los precios de los alimentos o a la falta transitoria de recursos naturales, por los desastres climáticos, sin caer en situaciones de conflicto. Esencialmente para esta autora hay dos factores importantes que explican cómo estas situaciones podrían dar lugar al conflicto: -cuando la inseguridad alimentaria se combina con otras tensiones sociales, como cuestiones de desempleo, pobreza, marginación social o falta de políticas de protección social; y -cuando los Estados no tienen instituciones suficientemente fuertes como para cumplir un pacto social con sus ciudadanos, proporcionando ayuda rápidamente a los ciudadanos afectados por la inseguridad alimentaria, con o sin asistencia internacional. Por tanto, para Cliffe la escasez de alimentos, por los motivos que sean, podría dar lugar al origen de conflictos cuando las estructuras del Estado sean débiles y/o deficientes en la protección de la seguridad alimentaria de la población.

Coincidimos con la postura de Cliffe ya que, por ejemplo, durante la crisis alimentaria de 2007 y 2008, debida al aumento del precio de los alimentos, los Estados en desarrollo con "baja fortaleza institucional" experimentaron más protestas por ese aumento que aquellos con "mayor fortaleza institucional", y muchas de estas protestas se tornaron violentas. Esto, por ejemplo, es la diferencia entre los eventos en Haití en comparación con aquellos en México o Filipinas, donde existía una mayor capacidad del Estado para lidiar con los choques de los precios de los alimentos y las protestas no estimularon el deterioro de la seguridad nacional o la violencia generalizada. A veces también leemos que la sequía que se produjo entre 2006 a 2009 fue un factor contribuyente al conflicto sirio, al impulsar la migración rural-urbana, que causó tensiones sociales. Entendemos que, por supuesto, este pudo haber sido un factor entre muchos, pero sería demasiado simplista sugerir que ese fue el principal impulsor del conflicto sirio.

Sin entrar en más consideraciones y tampoco hacer un análisis descriptivo de ejemplos concretos, coincidiendo con las conclusiones del estudio de Holleman, Jackson, Sánchez y $\operatorname{Vos}^{34}$, entendemos que la falta de alimentos o la inseguridad alimentaria pueden convertirse en un desencadenante de la violencia y la inestabilidad social, especialmente en contextos caracterizados por

${ }^{33}$ Cliffe, S., "Food security, Nutrition and Peace", discourse in the framework of Proceedings of The United Nations Security Council Meeting, FAO, New York, 29 March 2016, pp.21-23.

${ }^{34}$ Holleman, C., Jackson, J., Sánchez, M.v., Vos, R., (Eds), "Sowing the seeds of peace for food security. Disentangling the nexus between conflict, food security and peace", Economics Technical Study 2, FAO, Rome, 2017.

Araucaria. Revista Iberoamericana de Filosofia, Politica, Humanidades y Relaciones Internacionales, año $21, \mathrm{n}^{\circ} 42$. Segundo semestre de 2019. Pp. 157-182. ISSN 1575-6823 e-ISSN 2340-2199 doi: 10.12795/araucaria.2019.i42.08 
la desigualdad generalizada y las instituciones frágiles. Por tanto, compartimos la idea de que la falta de alimentos, motivada por diferentes factores, según el contexto social y económico, no es el único motivo de un conflicto, ya sea comunal, civil o interno, pero ayuda a contribuirlos, mantenerlos y/o intensificarlos. Por ejemplo, la inseguridad alimentaria puede actuar como un canal a través del cual se expresen agravios socioeconómicos y políticos más amplios. Es decir, estas quejas pueden verse agravadas por la desconfianza de la población hacia el gobierno, ante la sensación de falta de apoyo y respuesta del Estado cuando se produzcan períodos de inseguridad alimentaria ${ }^{35}$. Un ejemplo reciente de ello lo encontramos en Venezuela ${ }^{36}$. Podemos afirmar también que los aumentos repentinos en los precios de los alimentos tienden a exacerbar el riesgo de disturbios políticos y conflictos, sobre todo entre la población más pobre, como se vio en las crisis alimentarias de 2007-2008 cuando estallaron disturbios en más de 40 Estados $^{37}$.

Los eventos relacionados con el clima, especialmente las sequías, tienden a poner en peligro la seguridad alimentaria en términos de disponibilidad y acceso, lo que aumenta el riesgo de conflictividad social. Este es particularmente el caso donde existen profundas divisiones entre grupos de población o donde faltan mecanismos de resiliencia para mitigar o hacer frente a las adversidades climáticas. La competencia por los recursos naturales puede ser perjudicial para la seguridad alimentaria de los hogares rurales vulnerables, lo que puede culminar en conflictos ${ }^{38}$.

Es por ello que desde marzo de 2016 Graziano Da Silva, Director General de la FAO, instó al Consejo de Seguridad a poner de relieve la relación de interdependencia entre la seguridad alimentaria y el conflicto, así como su influencia en la desestabilización de las sociedades. La práctica del Consejo ha venido solicitando a la FAO que mantenga regularmente informados a sus miembros de la situación alimentaria en los Estados más críticos, sobre todo

35 Wischnath, G., Buhaug, H., "Rice or riots: on food production and conflict severity across India”, Political Geography, Volume 43, November 2014, pp. 6-15.

${ }^{36}$ Observatorio Venezolano de conflictividad social, "Conflictividad social en Venezuela en el primer trimestre de 2018”, 2018. Según este Informe del Observatorio, “de las 2.414 protestas registradas, 549 estuvieron vinculadas a la crisis alimentaria. Un promedio de seis protestas diarias por falta de alimentos". Véase también: El País, "Los venezolanos se acuestan con hambre", noticia en sección internacional, 22 de febrero de 2018, disponible en: https:/elpais.com/internacional/2018/02/22/ america/1519277784_904681.html, consultado el 20 de enero de 2019. Recientemente hemos podido apreciar en numerosos medios de comunicación cómo el régimen de Nicolás Maduro ha sumido al Estado de Venezuela en una grave crisis alimentaria en la que muchas personas carecen de alimentos y medicamentos básicos. A todo ello se han sumado otros problemas como la falta de energía eléctrica (apagones), los correspondientes saqueos y protestas civiles ante la grave situación y el bloqueo de la necesaria ayuda humanitaria para la población.

${ }^{37}$ FAO, FIDA, OMS, PMA y UNICEF, "El estado de la seguridad alimentaria y la nutrición en el mundo 2017, Fomentando la resiliencia en aras de la paz y la seguridad alimentaria”, FAO, Roma, 2017 , p.31.

${ }^{38}$ Hendrix, C., Brinkman, H.j., "Food Insecurity and Conflict Dynamics...Op.cit., p.3. 
desde la perspectiva de cómo la falta de seguridad alimentaria puede ser causa del impulso de conflictos ${ }^{39}$.

Ahora bien, a diferencia de los estudios que hemos analizado de la doctrina, que se centran en definitiva en considerar la falta de alimentos como un multiplicador o potenciador de las amenazas que puedan dar origen a un conflicto, ya sea comunal, civil o interno (que en eso estamos de acuerdo), consideramos también que en determinados casos la falta de alimentos, traducida en una hambruna generalizada, tanto en el interior de un Estado como entre Estados vecinos, puede llegar a ser de tal magnitud que en vez de considerar sólo a la falta de alimentos como un "multiplicador o potenciador de las amenazas" o como "multiplicador/potenciador de la aparición de conflictos", podríamos pensar que en sí es una amenaza que, unida a la vulnerabilidad concreta de la población, se convierta en un riesgo real. Por tanto, entendemos que no solo se debe considerar la falta de alimentos o la inseguridad alimentaria como un potenciador del riesgo o de la amenaza sino que en sí, dependiendo de su magnitud, podría ser considerada como un riesgo real. De hecho, ser un riesgo o una amenaza en sí mismo, no es lo mismo que ser un potenciador/multiplicador de riesgos y amenazas; existe una diferencia cualitativa al respecto.

Pudiera parecer que esto es poco probable pero ya desde el año 2003, en el marco de los asuntos que se relacionan con la responsabilidad del Consejo de Seguridad para el mantenimiento de la Paz y la Seguridad Internacionales, se examinó la cuestión de la crisis alimentaria de África como una amenaza para la paz y la seguridad ${ }^{40}$, aunque no existe ninguna Resolución al respecto que la califique como tal.

Asimismo, en la sesión en el Consejo de Seguridad que se celebró el 9 de agosto de 2017, en relación con el Mantenimiento de la Paz y la Seguridad Internacionales, el Consejo, declaró que:

"expresa su profunda preocupación [...] a la luz de la amenaza de hambruna sin precedentes que se cierne sobre Yemen, Somalia, Sudán del Sur y el noreste de Nigeria, que son zonas afectadas por conflictos"41.

Si nos fijamos, el Consejo de Seguridad de momento ya ha empleado la palabra "amenaza de hambruna" ${ }^{42}$. Por tanto, podríamos esperar que en

\footnotetext{
39 Para más información véase "Suplemento Paz y Seguridad Alimentaria", de 1 de mayo de 2016, disponible en: http://www.fao.org/3/a-i5638s.pdf. consultado el 21 de enero de 2019.

40 Disponible en: http://www.un.org/es/sc/repertoire/2000-2003/Chapter\%208/Thematic/0003_8_50B_Africa's $\% 20$ food $\% 20$ crisis $\% 20$ as $\% 20 \mathrm{a} \% 20$ threat $\% 20$ to $\% 20$ peace $\% 20$ and $\% 20$ security Spanish.pdf, p. 891, consultado el 21 de enero de 2019.

${ }^{41}$ UN Doc., S/PRST/2017/14, de 9 de agosto de 2017, p.1.

${ }^{42}$ En inglés emplea la expresión "the threat of famine". También emplea esta expresión, "amenaza de hambruna", en la Resolución 2417 (2018), de 24 de mayo de 2018, p.1.
} 
un futuro se incluyese la seguridad alimentaria en las responsabilidades del Consejo de Seguridad para el mantenimiento de la paz y seguridad internacionales, así como podríamos considerar la posibilidad de que este finalmente adopte una Resolución en la que califique expresamente a la falta de alimentos (entiéndase por hambruna, inseguridad alimentaria, crisis alimentarias severas, etc.) como una amenaza a la paz y seguridad internacionales.

Hay que tener en cuenta también, en este análisis, cómo el conflicto tiene asimismo un profundo impacto negativo sobre la seguridad alimentara, tanto directamente como indirectamente, tanto a medio como a largo plazo ${ }^{43}$.

Según el Índice Global del Hambre de 2017 (GHI, por sus siglas en inglés), elaborado por el Instituto Internacional de Investigación sobre Políticas Alimentarias (IFPRI), señala que los países con los niveles más altos de inseguridad alimentaria también son los más afectados por el conflicto $^{44}$. Además los Estados en situación de post-conflicto son un $40 \%$ más propensos a recaer en el conflicto, en un intervalo de tiempo de 10 años, si no se combaten los altos niveles de hambre ${ }^{45}$. Y es que, a menudo, los comportamientos violentos y el hambre están encerrados en círculos viciosos negativos en los que se "alimentan" el uno del otro ${ }^{46}$. Junto a otros factores, como los desastres climáticos o la pobreza, los conflictos son una de las principales causas de emergencia alimentaria que pueden dar lugar a la hambruna. Por dar más datos que revelen cómo el conflicto genera

${ }^{43}$ Por ejemplo, los conflictos pueden causar profundas recesiones económicas, impulsar la inflación y debilitar las finanzas públicas, lo que afecta a los medios de vida de las personas y, por ende, socava la seguridad alimentaria a largo plazo.

${ }^{44}$ IFPRI, "2017 global hunger index: The inequalities of hunger", disponible en: https://www.ifpri. org/publication/2017-global-hunger-index-inequalities-hunger consultado el 21 de enero de 2019.

${ }_{45}^{45}$ FAO, FAO, "Paz y seguridad alimentaria: invertir en la resiliencia para sostener los medios de vida rurales en situaciones de conflicto", nota técnica, Roma, 2016, p.16.

Estimaciones recientes sugieren que aproximadamente la mitad de los pobres del mundo viven hoy en países caracterizados por conflictos y violencia. Según el Informe de la FAO de marzo de 2017, "Perspectivas de cosechas y situación alimentaria", los 37 países que necesitan en la actualidad ayuda alimentaria externa son: Burkina Faso, Burundi, Camerún, República Centroafricana, Chad, Congo, Djibouti, Eritrea, Etiopía, Guinea, Haití, la República Popular Democrática de Corea, Iraq, Kenya, Lesotho, Liberia, Madagascar, Malawi, Malí, Mauritania, Mozambique, Myanmar, Nepal, Níger, Nigeria, Pakistán, Papúa Nueva Guinea, Sierra Leona, Somalia, Sudán del Sur, Sudán, Swazilandia, Yemen y Zimbabwe. De esos países la mayoría son africanos y presentan conflictos internos agravados por las consecuencias del cambio climático. En concreto, la delicada situación de la seguridad alimentaria en Sudán del Sur, un país empobrecido por decenios de guerra, se vio agravada por el estallido de la guerra civil en 2013. Actualmente, se calcula que 5,5 millones de personas padecen los niveles tres (crisis), cuatro (emergencia) y cinco (hambruna) de la Clasificación Integrada de la Seguridad Alimentaria en Fases (IPC por sus siglas en inglés). Véase: IPC Alert núm. 8, "In South Sudan, Food Insecurity Situation Still Dire And Widespread", 21 june 2017, disponible en: http://fscluster.org/sites/default/files/documents/ipc_alert_8_southsudan_may2017. pdf consultado el 20 de enero de 2019.

${ }^{46}$ Holleman, C., Jackson, J., Sánchez, M.v., Vos, R., (Eds), “Sowing the seeds...,Op.Cit., pp. 44 y ss.

Araucaria. Revista Iberoamericana de Filosofía, Política, Humanidades y Relaciones Internacionales, año $21, \mathrm{n}^{\circ} 42$. Segundo semestre de 2019. Pp. 157-182. ISSN 1575-6823 e-ISSN 2340-2199 doi: 10.12795/araucaria.2019.i42.08 
problemas de seguridad alimentaria ${ }^{47}$, en el Informe conjunto de la FAO y el PMA, sobre el "Monitoreo de la seguridad alimentaria en países con situaciones de conflicto", de 2017, se informa al Consejo de Seguridad sobre la débil seguridad alimentaria en 17 Estados actualmente en períodos de conflicto y post-conflicto. Siguiendo la Clasificación Integrada de la Seguridad Alimentaria en Fases, el citado informe muestra que los Estados con conflictos actuales tienen el mayor número de personas con inseguridad alimentaria. En algunos casos, estos porcentajes son alarmantes, incluyendo el 37\% del total de la población del sur de Sudán, el 50\% en Siria, y hasta el $89 \%$ de los refugiados sirios en el Líbano. Esta misma línea se mantiene en el mismo informe pero emitido en $2018^{48}$. En Sudán del Sur ahora, en 2018, se ha pasado al 45\%, y en Yemen se alcanza hasta el $60 \%$, es decir, más de la mitad de la población.

En general, podemos afirmar que la destrucción y la interrupción de la capacidad productiva del sistema alimentario, debido a los conflictos, donde ocurran, y la inseguridad civil derivada de ellos, socavan gravemente el acceso y la disponibilidad de alimentos, así como la salud y la nutrición de las personas, afectando por tanto de lleno a la seguridad alimentaria de la población. El impacto en los sistemas alimentarios en áreas predominantemente agrícolas puede ser grave y afectar a toda la cadena alimentaria: producción, cosecha, procesamiento, almacenamiento, transporte, financiación y comercialización de alimentos. En este sentido, el conflicto socava la resiliencia y, a menudo, obliga a las personas a desplazarse de sus hogares para buscar nuevos medios de subsistencia. En el caso de África, se identifica comúnmente que los conflictos, agravados por perturbaciones relacionadas con el clima, afectan considerablemente a la seguridad alimentaria y son, en gran parte, los causantes del reciente aumento de la inseguridad alimentaria ${ }^{49}$ en determinadas regiones, entre ellas Sudán del Sur, Nigeria, Somalia y Mali ${ }^{50}$.

${ }^{47}$ Para más información véase Cohen, M., Pinstrup-Andersen, P. "Food security and conflict", Social Research, 1999, p. 376; SIMMONS E., "Harvesting Peace: Food security, conflict, and cooperation", USAID, vol. 14, no 3, 2013, pp. 11 y ss.

${ }_{48}$ Disponible en: http://www.fao.org/3/I8386EN/i8386en.pdf consultado el 21 de enero de 2019.

${ }^{49}$ FAO, FIDA, OMS, PMA y UNICEF, "El estado de la seguridad alimentaria...2017, Op.Cit., p. ii.

${ }^{50}$ FAO, WFP, "Monitoring food security in countries with conflict situations: A joint FAO/WFP update for the United Nations Security Council”, 2018.

Araucaria. Revista Iberoamericana de Filosofia, Política, Humanidades y Relaciones Internacionales, año $21, \mathrm{n}^{\circ} 42$. Segundo semestre de 2019. Pp. 157-182. ISSN 1575-6823 e-ISSN 2340-2199 doi: 10.12795/araucaria.2019.i42.08 


\section{La dimensión alimentaria como factor sostenible de paz y seguridad internacionales}

Se ha establecido en los apartados anteriores que la paz y la seguridad internacionales, en la actual sociedad globalizada, incluye dimensiones diversas y, por tanto, su consecución no se basa simplemente en la ausencia de violencia. Contienen, además, entre otras dimensiones, la seguridad alimentaria, la seguridad medioambiental, la seguridad sanitaria, la regulación de los flujos masivos involuntarios de personas, la protección de los derechos humanos, etc. Todas estas dimensiones deben inscribirse en el marco de un concepto amplio de paz y seguridad internacionales ${ }^{51}$.

En lo relativo a la seguridad alimentaria, podemos decir que se constituye como un pilar importante de la paz y la seguridad internacionales, la estabilidad social y el desarrollo sostenible de los pueblos ${ }^{52}$. En la historia de la humanidad, se ha constatado continuamente el círculo negativo que vincula el hambre y la violencia y que genera conflictos e inestabilidad que pueden no estar circunscritos a las fronteras nacionales. Situaciones que en definitiva pueden poner en peligro la paz y seguridad internacionales o constituir una amenaza misma a estas.

En 2004, Naciones Unidas, en su Informe del Grupo de Alto Nivel sobre las amenazas y los desafíos internacionales, que lleva por título "Un mundo más seguro: la responsabilidad que compartimos" ${ }^{53}$, menciona seis grupos de amenazas que preocupan en el plano internacional, que están relacionadas entre sí, y deben afrontarse a nivel global, regional y nacional, dado que ningún Estado es inmune a las mismas. Estas son: Guerras entre Estados; Violencia dentro del Estado, con inclusión de guerras civiles, abusos en gran escala de los derechos humanos y genocidio; Pobreza, enfermedades infecciosas y degradación del medio ambiente; Armas nucleares, radiológicas, químicas y biológicas; Terrorismo; y Delincuencia transnacional organizada.

De entre este listado de amenazas, claramente las guerras entre Estados, la violencia dentro del Estado, la pobreza, la degradación del medio ambiente, así como el empleo de armas nucleares o químicas y el terrorismo repercuten negativamente en la seguridad alimentaria, incrementando un círculo negativo de inestabilidad en la población. Asimismo, la inseguridad alimentaria ${ }^{54}$ puede ser causa de conflictos internos, pobreza y del aumento de posibilidades de

${ }^{51}$ Véase Sotomayor Velásquez, A., "La seguridad internacional: vino viejo en botellas nuevas", Revista de ciencia politica, Santiago, vol. 27, no 2, 2007, p. 67-88.

52 En concreto el Objetivo 2 de la Agenda 2030 para el Desarrollo Sostenible pretende "poner fin al hambre, lograr la seguridad alimentaria y promover la agricultura sostenible". Véase UN. Doc A/69/L.85, de 12 de agosto de 2015, p.17.

${ }^{53}$ UN. Doc A/59/565, 2 de diciembre de 2004.

54 Véase: FAO, "Crop Prospects and Food situation", Quarterly Global Report, Roma, junio 2017. 
contraer enfermedades infecciosas al hacer a las personas más vulnerables y con menor resistencia inmunológica.

En este sentido, la protección de la seguridad alimentaria puede utilizarse como una herramienta operacional tanto de prevención, como de mitigación o estabilización de las amenazas internacionale ${ }^{55}$, sobre todo en aquellas regiones en vías de desarrollo donde el sector agrícola y ganadero desempeñen un papel fundamental como medio económico y social de vida de la población ${ }^{56}$.

En general, el inicio de una crisis o conflicto no se produce debido a un sólo motivo o factor, ya que normalmente las amenazas a la paz y seguridad internacionales se interconectan y agravan su peligrosidad ${ }^{57}$. Por ejemplo, la falta de alimentos, salvo en situaciones extremas, no es el único motivo de la crisis, el conflicto, o la emergencia de una amenaza, pero puede ayudar a contribuirlos y agravar sus consecuencias, impidiendo crear las condiciones de una paz duradera. A su vez, a la falta de alimentos puede verse agravada por otros factores, como el cambio climático, o la pobreza, haciendo que la respuesta frente a los nuevos desafíos y amenazas deba de tener en cuenta el contexto general y una dimensión holística y multidimensional.

Por tanto, podemos decir que la seguridad alimentaria queda mermada o quebrantada por el impacto que provocan sobre ella ciertas amenazas a la paz y seguridad internacionales y que a su vez, la falta de seguridad alimentaria puede exacerbar la peligrosidad de otras amenazas actuales a la paz y seguridad internacionales o incluso puede acabar dando origen a alguna de ellas. Podríamos en este sentido, situar la falta de seguridad alimentaria como una amenaza emergente ${ }^{58}$, teniendo en cuenta que el hambre en el mundo ha aumentado en los últimos años.

Sin embargo, actualmente, no contamos con ninguna Resolución del Consejo de Seguridad que califique, de acuerdo con el artículo 39 de la Carta (Capítulo VII), a la falta de alimentos o a la inseguridad alimentaria como una amenaza a la paz y seguridad internacionales, lo cual supondría la viabilidad

${ }_{55}$ Coincidiendo con alguno de los objetivos de las Operaciones de Mantenimiento de la Paz. Según el Profesor FERNÁNDEZ SÁNCHEZ, el objetivo esencial que persiguen estas operaciones es el "despliegue preventivo con el que se pretende aliviar los sufrimientos y limitar o controlar la violencia, reducir riesgos en zonas fronterizas o evitar agresiones”. Véase FERNÁNDEZ SÁNCHEZ, P. A., Operaciones.., Op.Cit., p.30.

${ }_{56}$ FAO, "Paz y Seguridad Alimentaria: invertir en..., Op.Cit., p.2.

${ }^{57}$ Mayor Zaragoza, F., Ackerman, M., Mesa, M., Álvarez-Ossorio, I., Ríos, X., Saurina, M., "Seguridad internacional y democracia: guerras, militarización y fronteras", Anuario CEIPAZ, Fundación Cultura de Paz, Madrid, 2017, p.32.

${ }^{58}$ De hecho el Secretario General califica la Inseguridad Alimentaria como "una cuestión emergente de alcance mundial". Véase: UN. Doc, A/71/283, 3 de agosto de 2016, p.7. Asimismo, en la Carta de envío, de fecha 1 de diciembre de 2004, dirigida al Secretario General por el Presidente del Grupo de alto nivel sobre las amenazas, los desafíos y el cambio, en el apartado "las amenazas con que nos enfrentamos", establece que "las tendencias actuales indican que en muchos países, especialmente en el África subsahariana, persiste y posiblemente se esté agravando la inseguridad alimentaria”. Véase: UN Doc. A/59/565, 2 de diciembre de 2004, para.52. 
de activar sus competencias en virtud del Capítulo VII de la Carta y, por tanto, de adoptar medidas obligatorias en este ámbito. Si bien es cierto que esta cuestión está asumiendo cada vez más importancia entre las preocupaciones del Consejo ${ }^{59}$, el cual ha solicitado a la FAO y al PMA información periódica, desde 2016, sobre la situación de la seguridad alimentaria en los Estados afectados por conflictos ${ }^{60}$.

Asimismo, dado que la paz no es la simple ausencia de violencia, como se ha dicho reiteradamente, sino que requiere además de medidas y acciones que la consoliden a largo plazo, es coherente argumentar que la protección de la seguridad alimentaria es un complemento esencial, que no único, de la consolidación de la paz y por tanto cabe situarla como factor sostenible de paz y seguridad internacionales. En parecidos términos, Mahmoud, Connolly y Mechoulan entienden que "la paz positiva requiere construir y fortalecer los factores que la fomentan [...]; entre estos factores están los que permiten la paz cotidiana [...] y otros factores más sistémicos como el acceso a los alimentos y al agua potable, la distribución equitativa de los recursos, el buen funcionamiento del aparato institucional, etc." ${ }^{\prime 1}$.

En este sentido, no resulta osado afirmar que sería un complemento esencial de las acciones o medidas de consolidación de la paz el restablecimiento de la seguridad alimentaria o el fortalecimiento de la misma en situaciones de conflictos o crisis prolongadas. Estas ideas, de hecho, han sido también asumidas también por el Comité Mundial de la Seguridad Alimentaria o la actual Relatora Especial de Naciones Unidas para el Derecho a la Alimentación, como veremos a continuación.

Asimismo, la FAO, la OMS, o UNICEF relacionan la protección de la seguridad como factor sostenible de paz. Prueba de ello es el Informe que publicaron conjuntamente en 2017, titulado "El estado de la seguridad alimentaria y la nutrición en el mundo 2017: Fomentando la resiliencia en aras de la paz y la seguridad alimentaria" ${ }^{2}$. En este Informe, se hace un análisis de la importancia de la seguridad alimentaria en el mantenimiento de la paz,

\footnotetext{
${ }^{59}$ Veáse por ejemplo "Repertorio de la práctica seguida por el Consejo de Seguridad: La amenaza de la crisis alimentaria de África para la paz y la seguridad", 2005, disponible en: http://www.un.org/ es/sc/repertoire/2004-2007/Chapter8/Thematic/04-07_8_Africa'sfoodcrisisasathreattopeaceandsecur ity.pdf., consultado el 22 de enero de 2019.

${ }^{60}$ La propuesta de reunión con el Consejo de Seguridad para tratar estos temas fue iniciada por la República de Angola ante Naciones Unidas y la Misión Permanente de España ante Naciones Unidas, que convocaron el 29 de marzo de 2016 la reunión ante el Consejo. La reunión se realizó con arreglo a la "Fórmula Arria". Esta fórmula es una práctica reciente de los miembros del Consejo de Seguridad bajo la cual estos pueden intercambiar impresiones y opiniones directamente, sobre un tema comprendido en el ámbito de responsabilidad del Consejo de Seguridad, con los altos representantes de los gobiernos y las organizaciones internacionales, a menudo previa solicitud de estos últimos.

${ }^{61}$ Mahmoud, Y., Connolly, L., Mechoulan, D., "Sustaining Peace in Practice: Building on What Works", International Peace Institut, 2018, p.8.

${ }^{62}$ FAO, IFAD, UNICEF, WFP and WHO, "The State of Food Security and Nutrition in the World 2017. Building resilience for peace and food security”, Rome, 2017.
} 
considerando, entre otros aspectos, que las intervenciones oportunas que tengan en cuenta las situaciones del conflicto y cuya finalidad sea mejorar la seguridad alimentaria y la nutrición, pueden ayudar a mantener la paz.

Siguiendo esta misma línea, la actual Relatora Especial de Naciones Unidas para el Derecho a la Alimentación, en uno de sus últimos Informes oficiales hace referencia a esta vinculación, al afirmar que "la eliminación del hambre y la malnutrición es indispensable para una paz mundial sostenible"63.

Sin embargo, la interrelación entre la paz y la seguridad alimentaria ya fue establecida en 2015 por el Comité de la Seguridad Alimentaria Mundial en el documento que elaboró sobre el "Marco de Acción para la Seguridad Alimentaria y la Nutrición en Crisis Prolongadas" ${ }^{64}$. Este Marco de Acción fue aprobado por los Estados miembros de la FAO en octubre de 2015. Si bien es voluntario y no vinculante, el Marco se basa en instrumentos internacionales y regionales y marcos mundiales que gozan de amplio respaldo, incluidos los Objetivos de Desarrollo Sostenible. Fue la primera vez que se articuló un consenso mundial para mitigar las amenazas a la seguridad alimentaria y la nutrición en crisis prolongadas. Concretamente, el documento se basa en 11 principios, dentro de los cuales el principio 9 hace referencia expresa a "contribuir a la consolidación de la paz a través de la seguridad alimentaria" 65 .

De hecho, la interrelación entre la seguridad alimentaria, el desarrollo y la paz en definitiva es una cuestión que se aprecia en los Objetivos de Desarrollo Sostenible (ODS) elaborados por la Asamblea General, que tienen la finalidad de fortalecer la paz universal dentro de un concepto más amplio de la libertad en el entendido de que "no puede haber desarrollo sostenible sin paz, ni paz sin desarrollo sostenible". En este sentido, en la declaración de su Resolución 70/1, de 2015, establece que "la construcción de sociedades pacíficas, justas e inclusivas requiere poner fin a la pobreza y el hambre en todo el mundo de aquí a $2030 " 66$.

\subsection{La protección de la seguridad alimentaria en el marco estratégico del sostenimiento de la paz de Naciones Unidas}

Tras la lectura de diferentes estudios de la doctrina y de los informes de organismos especializados y programas de Naciones unidas, podemos concluir que si bien la falta de alimentos, o en más amplitud la inseguridad

${ }^{63}$ UN Doc. A/HRC/37/61, de 25 de enero de 2018, Informe de la Relatora Especial sobre el derecho a la alimentación sobre la Promoción y protección de todos los derechos humanos, civiles, políticos, económicos, sociales y culturales, incluido el derecho al desarrollo, 2018, para.5, p.3.

64 Véase CSA "Marco de Acción para la Seguridad Alimentaria y la Nutrición en Crisis Prolongadas", CSA, 13 octubre de 2015, disponible en: http://www.fao.org/3/a-bc852s.pdf, consultado el 22 de enero de 2018.

${ }^{65}$ Ibidem, p. 20.

${ }^{66}$ UN. Doc., A/RES/70/1, 21 de octubre de 2015, p.3. 
alimentaria, no es la única causa de un conflicto, está claro que ayuda a iniciarlos y que los puede mantener y/o intensificar. Cuando la inseguridad alimentaria se combina con otras tensiones, como el desempleo, la pobreza, la marginación social o falta de políticas de protección social, los desastres climáticos, etc., entonces sí que la falta de alimentos podría ser, por explicarlo comúnmente, como "la gota que colma el vaso" ${ }^{67}$. Esto es, un motivo clave para la movilización de la gente, que puede dar lugar tanto a protestas no violentas como violentas, o empujar a participar en conflictos armados ${ }^{68}$. Podemos apreciar también esto en Siria, donde la sequía, el desplome de la producción de alimentos y el deterioro de los medios de vida de la población, actuaron como un catalizador de la crisis actual.

Entendíamos también, en los apartados anteriores, que en determinados casos, la hambruna generalizada puede llegar a ser una amenaza en sí, aunque esta opinión no ha sido contemplada por la doctrina. El hecho de que actualmente más de la mitad de la población de Yemen padezca de inseguridad alimentaria, con un alto grado de emergencia según la clasificación integrada de la seguridad alimentaria en fases ${ }^{69}$, nos hace repensar sobre esta idea.

Por tanto, el razonamiento es el siguiente: si la inseguridad alimentaria es un multiplicador de las amenazas para el conflicto, o en determinados casos de hambruna generalizada puede llegar a ser una amenaza en sí, podría pensarse que la mejora de la seguridad alimentaria puede reducir las tensiones, contribuir a crear entornos más estables o impedir en cierta medida la aparición de conflictos. Si estas intervenciones se llevan a cabo correctamente, el círculo vicioso negativo entre la falta de alimentos y el conflicto puede transformarse en un ciclo virtuoso positivo de seguridad alimentaria y estabilidad que proporcione dividendos de la $\mathrm{paz}^{70}$, reduzca los factores de conflicto y mejore la cohesión social. De hecho, según Collier, Hoeffler y Söderbom, las situaciones posteriores a los conflictos suelen ser frágiles, con un $40 \%$ volviendo al

${ }^{67}$ Bonnecase, V., "Revueltas del hambre", en Collart Dutilleul, F. (Ed.), Bugnicourt, J. F., (Dir.) Diccionario Jurídico de la Seguridad Alimentaria en el Mundo, Monografía jurídica, INIDA, Costa Rica, 2014, pp.490-495.

${ }^{68}$ Simmons, E., "Harvesting Peace...,Op.Cit., p.23.

${ }^{69}$ FAO, WFP, "Monitoring food security in countries with conflict situations...,Op.Cit, p.39.

${ }^{70}$ Se trata de servicios administrativos y sociales ofrecidos como dividendos de la paz, enmarcados en las estrategias de consolidación de paz, con el fin de reducir las tensiones sociales mediante la provisión de servicios tangibles y necesarios, creando incentivos para evitar el comportamiento violento y apoyando los esfuerzos de construcción del Estado en momentos críticos del proceso de paz. Por ejemplo, el PMA apoyó la creación de programas sectoriales de protección de la seguridad alimentaria en Sudán del Sur y Nepal para promover desarrollo y ayudar a las instituciones nacionales a hacer frente a los diferentes factores del conflicto. En Sudán del Sur se apoyó la reserva de cereales y en Nepal se creó un sistema de monitoreo de la volatilidad de los precios de los alimentos para ayudar al gobierno a responder cuando estos subían. Véase: Mccandless, E., "Peace Dividends and Beyond: Contributions of Adminstrative and Social Services to Peacebuilding”, United Nations Peacebuilding Support Office, 2012, pp. 3, 6. 
conflicto en los diez años siguientes ${ }^{71}$, por lo que lo lógico es que se aborden las causas profundas de los conflictos que dan lugar a su origen, para que éstos no reaparezcan.

En el sentido que hemos expresando, la Oficina de Consolidación de la Paz de Naciones Unidas considera que el Fondo de Consolidación de la paz debería apoyar "processes to ensure a peacebuilding lens in poverty and food security"72.

Esta perspectiva se refuerza más recientemente en las Resoluciones del Consejo de Seguridad y la Asamblea General, de abril de 2016, sobre la consolidación de la $\mathrm{paz}^{73}$, donde se introdujo el concepto de "sostenimiento de la paz" (sustaining peace), como un marco unificador para abordar las causas profundas del conflicto. En estas Resoluciones se destaca la importancia de que se adopte un enfoque amplio para sostener la paz por parte de Naciones Unidas, en particular previniendo los conflictos y abordando sus causas profundas, mediante el crecimiento económico sostenido y sostenible, la erradicación de la pobreza, el desarrollo social, y el desarrollo sostenible, entre otros fines ${ }^{74}$. Como es sabido, la protección de la seguridad alimentaria está íntimamente relacionada con la erradicación de la pobreza, el desarrollo social ${ }^{75}$ y el crecimiento económico local ${ }^{76}$. De este modo, atendiendo a las Resoluciones conjuntas de la Asamblea y del Consejo en relación con el sostenimiento de la paz, de 2016, la protección de la seguridad alimentaria debe ser objeto de incorporación, como prioridad estratégica, en las estrategias que se lleven a cabo en este marco.

Por tanto, dentro de este marco estratégico de Naciones Unidas para el sostenimiento de la paz, cabría el apoyo de la seguridad alimentaria y a los medios de producción de alimentos (agricultura, ganadería, pesca) a fin de abordar no solo los síntomas sino también las causas profundas del conflicto y también a modo de prevenirlo.

En este sentido, las resoluciones y recomendaciones recientes en el marco del sistema de Naciones Unidas, incluidos la adopción de los Objetivos de Desarrollo Sostenible y la revisión de la arquitectura de Consolidación de la Paz, comparten un mensaje común sobre la interrelación entre paz, seguridad y desarrollo. Empieza a considerarse, pues, que Naciones Unidas quiere asumir

\footnotetext{
${ }^{71}$ Collier, P., Hoeffler, A., Söderbom, M., "Post-conflict risks", Journal of Peace Research, Volume: 45 issue: 4, 2008, pp. 461-463.

72 United Nations Peacebuilding Support Office "Peace Dividends and Beyond: Contributions of Adminstrative and Social Services to Peacebuilding", 2012, p.6.

73 UN.Doc., S/RES/2282 (2016)*, 27 de abril de 2016; A/RES/70/262, sobre Examen de la Estructura de las Naciones Unidas para la Consolidación de la Paz.

${ }^{74}$ Ibidem, p.2.

75 Véase: Hong, P., "Peace and Stability as Enablers for and Outcome of Development", United Nations Department of Economic and Social Affairs (DESA), 2015.

${ }^{76}$ Hendrix, C., Brinkman, H. J., "Food Insecurity and Conflict...,Op.cit., p.9.
} 
un nuevo compromiso colectivo para promover la paz sostenible y evitar los conflictos, valiéndose de los diferentes objetivos de su mandato y labor, y ciñéndose a ellos. En este planteamiento novedoso se intuye (a veces de forma expresa y otras veces de forma implícita), por un lado, la importancia de comprender mejor las relaciones existentes entre la seguridad alimentaria, la paz y la prevención de los conflictos, y, por otro, la forma en que la reducción de los riesgos y el fomento de la resiliencia mediante la seguridad alimentaria pueden contribuir a mantener una paz sostenible. En definitiva, podemos considerar que esto da lugar a que la seguridad alimentaria se constituya como un factor sostenible de paz.

Cada vez hay más pruebas de que, cuando se aplican adecuadamente, las intervenciones oportunas relacionadas con la seguridad alimentaria pueden generar resiliencia al conflicto, no solo ayudando a los Estados y a las personas a sobrellevar el conflicto y recuperarse de él, sino también contribuyendo a la prevención ${ }^{77}$ y mitigación. Si se protege la seguridad alimentaria, se fomenta el desarrollo, la cohesión social y se lucha contra la pobreza, factores estos que contribuyen a la prevención y/o mitigación de los conflictos y, en consecuencia, apoyan los esfuerzos del "sostenimiento de la paz".

Los esfuerzos por reactivar el sector agrícola y mejorar la seguridad alimentaria, incluso a través de la protección social, tienen efectos positivos en la sostenibilidad de la paz. Son importantes "dividendos de la paz". Esto lo reconoce, por ejemplo, el Gobierno de Colombia, que considera la agricultura, el desarrollo rural y la seguridad alimentaria como piedras angulares del proceso de paz y de la cohesión social que debe construirse en la fase posterior al conflicto ${ }^{78}$.

\section{Conclusiones}

La seguridad alimentaria es un pilar de la paz y la seguridad internacionales en la medida en que contribuye a prevenir, mitigar o estabilizar los conflictos y a su vez la falta de alimentos puede contribuir a iniciarlos junto a otros factores. Por tanto, sería conveniente añadir el indicador de "falta de alimentos" en los sistemas de alerta temprana de los conflictos.

77 Breisinger, C., Ecker, O., Maystadt, J.f., Trinh Tan, J. F., Al-Riffai P., Bouzar, K., Sma A., Abdelgadir, M., "How to build resilience to conflict: The role of food security", IFPRI Food Policy Report, Washington, 2014, p.17.

${ }_{78}$ El gobierno de Colombia y las FARC han solicitado a la FAO acompañar la implementación del primer punto del acuerdo de paz, el cual está centrado en la lucha contra el hambre, el desarrollo del campo y una reforma rural integral. El acuerdo de paz es fruto de una negociación de cuatro años y pone fin a cinco décadas de conflicto armado. El primer punto del acuerdo "Hacia un Nuevo Campo Colombiano: Reforma Rural Integral", busca generar una transformación en las zonas rurales de Colombia, duramente golpeadas por el conflicto armado. Información disponible en: FAO, "Colombia pide a FAO acompañar la implementación de los acuerdos de paz”, Noticias, 30 agosto 2016.

Araucaria. Revista Iberoamericana de Filosofía, Política, Humanidades y Relaciones Internacionales, año $21, \mathrm{n}^{\circ} 42$. Segundo semestre de 2019. Pp. 157-182. ISSN 1575-6823 e-ISSN 2340-2199 doi: 10.12795/araucaria.2019.i42.08 
Si entendemos que la seguridad ha tomadoun carácter multidimensional (que incluye aspectos militares, políticos, económicos, sociales, medioambientales y humanitarios), resulta adecuado decir que la seguridad alimentaria tiene que tomarse en consideración a la hora de abordar las llamadas Estrategias de Seguridad. Esto es, en la búsqueda de soluciones al problema de la inseguridad, los Estados deben afrontar los aspectos de la seguridad alimentaria, a modo de prevenir crisis alimentarias (que pueden estar producidas por circunstancias que escapan a su control, como las tendencias económicas del mercado, el aumento demográfico, las adversidades climáticas, los desplazamientos de población, etc.), en consonancia con los Objetivos 2 y 16 de Desarrollo Sostenible de Naciones Unidas.

Si bien la falta de alimentos no es el único factor que puede generar conflictos, está claro que contribuye a iniciarlos y/o exacerbarlos. El hambre produce inestabilidad social cuando las personas no disponen de alimentos y tienen que tomar decisiones por desesperación. Esto se manifiesta de diversas maneras, a veces en los disturbios sociales, las protestas, la falta de confianza en el Estado, otras veces en conductas violentas a través del saqueo, robo, o el apoyo y/o reclutamiento de movimientos violentos o grupos armados que ofrecen seguridad y alivio alimentario a corto plazo a cambio de lealtad hacia sus ideologías depravadas.

En este sentido, un contexto en el que exista seguridad alimentaria ofrecerá mayores posibilidades, junto a otros factores, de configurarse como un marco sostenible de paz. Reforzar la protección de la seguridad alimentaria en aquellos lugares inmersos en crisis o conflictos, como una medida de consolidación de la paz, revierte positivamente en el respeto de los derechos humanos y en el desarrollo, por lo que genera dividendos de paz y por tanto contribuye a crear una paz sostenible.

Naciones Unidas quiere asumir un nuevo compromiso colectivo para promover la paz sostenible y evitar los conflictos, amparándose jurídicamente en los diferentes objetivos de su mandato y labor, y ciñéndose a ellos. En este marco de sostenibilidad de la paz se puede apreciar, en ocasiones de forma expresa y otras veces de manera implícita, por un lado, la importancia de comprender mejor las interrelaciones existentes entre la seguridad alimentaria, la paz y la prevención de los conflictos, y, por otro lado, la forma en que la reducción de los riesgos y el fomento de la resiliencia mediante la seguridad alimentaria pueden contribuir a mantener una paz sostenible. Podemos pensar que esto da lugar a que la seguridad alimentaria sea considerada, en el marco de Naciones Unidas, como un factor sostenible de paz, por lo que debe ser objeto de incorporación, como prioridad estratégica, en las estrategias de sostenimiento de la paz que se lleven a cabo. 


\section{Referencias bibliográficas:}

Abellán Honrubia, V., "Sobre el método y los conceptos del Derecho Internacional Público", en Soberanía del Estado y Derecho Internacional: Homenaje al Profesor Juan Antonio Carrillo Salcedo (O.C.), primer volumen, Universidad de Sevilla, Secretariado de Publicaciones, 2005.

Afifi, A., "Sources et norms de sécurité alimentaire en Droit International", en Mahiou, A., and Snyder, F., (Ed.), Food Security and Food Safety/ La Sécurité Alimentaire, Martinus Nijhoff Publishers, Dordrecht, Netherlands, 2006.

Ayala, A., "A human rights approach to the health implications of food and nutrition insecurity", Public Health Reviews, 38:10, 2017.

Barbé, E., Relaciones Internacionales, Tecnos, Madrid, 1995.

Blattman, C., Miguel, E., "Civil war", Journal of Economic Literature, 48(1), 2010.

Bonnecase, V., "Revueltas del hambre", en Collart Dutilleul, F. (Ed.), BUGNICOURT J. F., (Dir.) Diccionario Jurídico de la Seguridad Alimentaria en el Mundo, Monografía jurídica, Inida, Costa Rica, 2014.

Boyd Orr, J., "Science, Politics and Peace", Lecture delivered on the occasion of the award of the Nobel Peace Prize of 1949.

Breisinger, C. et al., "How to build resilience to conflict: The role of food security”, IFPRI Food Policy Report, Washington, 2014.

Breisinger, C., Ecker, O., Maystadt, J.f., Trinh Tan, J. F., Al-Riffai, P., Bouzar, K., Sma, A., Abdelgadir, M., "How to build resilience to conflict: The role of food security", IFPRI Food Policy Report, Washington, 2014.

Buzan, B., "New Patterns of Global Security in the Twenty-First Century", International Affairs, Vol. 67, №3, 1991.

Cliffe, S., "Food security, Nutrition and Peace", discourse in the framework of Proceedings of The United Nations Security Council Meeting, FAO, New York, 29 March 2016.

Cohen, M., Pinstrup-Andersen, P. "Food security and conflict”, Social Research, 1999.

Collier, P., Hoeffler, A., Söderbom, M., "Post-conflict risks", Journal of Peace Research, Volume: 45 issue: 4, 2008.

Del Arenal, C., "El nuevo escenario mundial y la teoría de las relaciones internacionales", en Pérez González, M. (coord.), Hacia un nuevo orden internacional y europeo: estudios en homenaje al profesor don Manuel Díez de Velasco, Tecnos, Madrid, 1993.

FAO, "Colombia pide a FAO acompañar la implementación de los acuerdos de paz”, Noticias, 30 agosto 2016. 
FAO, "Crop Prospects and Food situation", Quarterly Global Report, Roma, junio 2017.

FAO, "Paz y seguridad alimentaria: invertir en la resiliencia para sostener los medios de vida rurales en situaciones de conflicto", nota técnica, Roma, 2016.

FAO, FIDA, OMS, PMA y UNICEF, "El estado de la seguridad alimentaria y la nutrición en el mundo 2017, Fomentando la resiliencia en aras de la paz y la seguridad alimentaria", FAO, Roma, 2017.

FAO, WFP, "Monitoring food security in countries with conflict situations: A joint FAO/WFP update for the United Nations Security Council", 2018.

Fernández Sánchez, P.A., Operaciones de Mantenimiento de la Paz (Vol.I), Análisis Jurídico de las Operaciones de Mantenimiento de la Paz, Ministerio de Educación y Cultura, Universidad de Huelva, Madrid, 1998.

Giles Carnero, R., "El cambio climático como riesgo y amenaza para la seguridad: derivaciones en el desarrollo del régimen jurídico internacional en materia de clima", Araucaria, vol. 18, núm. 36, 2016.

Hendrix, C., Brinkman, H.j., "Food Insecurity and Conflict Dynamics: Causal Linkages and Complex Feedbacks", Stability: International Journal of Security and Development, 2(2), 2013.

Hirshleifer, J., The Dark Side of the Force: Economic Foundations of Conflict Theory, Cambridge, Cambridge University Press, 2001.

Holleman, C., Jackson, J., Sánchez, M.v., Vos, R., (Eds), "Sowing the seeds of peace for food security. Disentangling the nexus between conflict, food security and peace", Economics Technical Study 2, FAO, Rome, 2017.

Hong, P., "Peace and Stability as Enablers for and Outcome of Development", United Nations Department of Economic and Social Affairs (DESA), 2015.

Laborie Iglesias, M, "La Evolución del Concepto de Seguridad", Documento Marco 05/2011, Instituto Español de Estudios Estratégicos, Ministerio de Defensa, 2011.

Madrueño-Aguilar, R., "Human Security and the New Global Threats: Discourse, Taxonomy and Implications", Global Policy, vol. 7, no 2, 2016.

Mahmoud, Y., Connolly, L., Mechoulan, D., "Sustaining Peace in Practice: Building on What Works", International Peace Institut, 2018.

Mayor Zaragoza, F., Ackerman, M., Mesa, M., Álvarez-Ossorio, I., Ríos, X., Saurina, M., "Seguridad internacional y democracia: guerras, militarización y fronteras", Anuario CEIPAZ, Fundación Cultura de Paz, Madrid, 2017.

Mccandless, E., "Peace Dividends and Beyond: Contributions of Adminstrative and Social Services to Peacebuilding", United Nations Peacebuilding Support Office, 2012. 
Morán Blanco, S. "La seguridad medioambiental y el cambio climático: consecuencias a nivel internacional", en Azeredo Lopes, J.a., Fernández Sánchez, P.A., Seguridad Medioambiental y Orden Internacional, Atelier, Barcelona, 2015.

Observatorio Venezolano de conflictividad social, "Conflictividad social en Venezuela en el primer trimestre de 2018”, 2018.

Pinstrup-Andersen, P., Shimokawa, S., "Do poverty and poor health and nutrition increase the risk of armed conflict onset?", Food Policy, 33(6), 2008.

Reno, W., Warfare in independent Africa, Cambridge University Press, New York, pp.2-30, 2011.

Simmons, E., "Harvesting Peace: Food security, conflict, and cooperation", USAID, vol. 14, no 3, 2013.

Sotomayor Velásquez, A., "La seguridad internacional: vino viejo en botellas nuevas", Revista de ciencia política, Santiago, vol. 27, no 2, 2007.

Toucoz, J., "Mondialisation et sécurité économique internationale. Quelques remarques juridiques et institutionnelles", $R G D I P, \mathrm{n}^{\circ} 3$.

UN Doc. A/HRC/37/61, de 25 de enero de 2018.

UN Doc. A/RES/70/1, 21 de octubre de 2015, "Transformar nuestro mundo: la Agenda 2030 para el Desarrollo Sostenible".

UN Doc., A/RES/72/173, 26 de enero de 2018.

UN Doc., S/PRST/2017/14, de 9 de agosto de 2017.

UN Doc., S/RES/2282 (2016)*, de 27 de abril de 2016.

UN. Doc A/59/565, 2 de diciembre de 2004.

UN. Doc, A/71/283, 3 de agosto de 2016.

UN.Doc, A/RES/377 (V), Unión pro paz, de 3 de noviembre de 1950.

UN.Doc. S/PV.7389, 23 February 2015.

UN.Doc., S/23500, 31 enero 1992.

UN.Doc., S/RES/2282 (2016)*, 27 de abril de 2016; A/RES/70/262, sobre Examen de la Estructura de las Naciones Unidas para la Consolidación de la Paz.

Uribe Vargas, D., El Derecho a la Paz, Facultad de Derecho de la Universidad Nacional de Colombia, Instituto para el desarrollo de la Democracia, Bogotá, 1996.

Wischnath, G., Buhaug, H., "Rice or riots: on food production and conflict severity across India”, Political Geography, Volume 43, November 2014. 